\title{
Economic agents as imperfect problem solvers
}

\section{PRELIMINARY AND INCOMPLETE - PLEASE DO NOT CIRCULATE}

\author{
Cosmin Ilut \\ Rosen Valchev \\ Duke \& NBER \\ Boston College
}

February 2017

\begin{abstract}
In this paper we study a bounded rationality model where agents find it costly to solve economic problems, even if state variables are perfectly observed. We analyze how this hypothesis is complementary, but distinct, from the traditional approach of "information constraints', where agents are limited in the proper perception of state variables. We consider several classic economic problems, with a particular focus on macroeconomic models, to derive observable implications that differentiate our proposed friction from the existing bounded rationality approaches. The model generates state-dependent decision rules characterized by inaction, as well as by under- or over-reaction. In terms of policy implications, we show how the cognition friction can change inference on the underlying sources of economic mechanisms and shocks.
\end{abstract}

